Теорія Ймовір. та Матем. Статист. Вип. 82, 2010
Theor. Probability and Math. Statist.

No. 82, 2011, Pages 171-175

S 0094-9000(2011)00836-8

Article electronically published on August 5, 2011

\title{
ON A CONSTANT RELATED TO AMERICAN TYPE OPTIONS
} UDC 519.21

\author{
GEORGIĬ SHEVCHENKO
}

\begin{abstract}
We discuss a constant which arises in several problems related to optimal exercise of American derivative securities.
\end{abstract}

\section{INTRODUCTION}

In the paper [ $[$, we have encountered an interesting constant. More precisely, we have considered the following problem in [6]: determine a stopping time $\tau \in[0, T]$ such that the mathematical expectation E $\left[S_{\tau}^{1}-S_{\tau}^{2}\right]$ is maximal, where $S_{t}^{1}$ and $S_{t}^{2}$ are correlated geometric Brownian motions. This problem can be viewed as a problem on the optimal exercise time to exchange two assets. It is shown in $[6$ that the optimal exchange moment is the first time when a certain increasing function $\beta(t)$ exceeds the ratio $S_{t}^{1} / S_{t}^{2}$, and that this "threshold" function satisfies the following equation:

$$
\beta(T-t)=\frac{\delta_{1}}{\delta_{2}}\left[1-\sigma a(T-t)^{1 / 2}+O(T-t)\right], \quad t \rightarrow T-,
$$

where $\delta_{i}$ is a drift coefficient for $S_{t}^{i}, \sigma$ is the volatility for $S_{1}(t) / S_{2}(t)$, and the constant $a$ is a solution of a certain complicated equation (we will present this equation later in this paper). In the same paper [6], another characterization of the constant $a$ is obtained; namely, $a$ is a solution of the equation

$$
a=\sup _{\tau \in[0,1]} \frac{\mathrm{E}\left[\tau W_{\tau}\right]}{\mathrm{E}[\tau]}
$$

where $W$ is the standard Brownian motion and where the supremum is taken over the set of all stopping times.

Later we found other papers where this constant is discussed. For example, the constant is used in [8, 3, 5] in the problem of an optimal exercise for an American option on a dividend-paying stock and, in 4, for the problem of optimal exercise of delayed-exercise options. In some papers, say in [8, 2, 4, this constant is written in a different form, $a \sqrt{2}$ instead of $a$; however, its value is the same, since the underlying term of the asymptotic expansion in our studies is $a \sigma(T-t)^{1 / 2}$, while the corresponding term in [8, 2, 4] is $c \sigma\left(\frac{1}{2}(T-t)\right)^{1 / 2}$. A similar reason explains why $a / \sqrt{2}$ is used in [3] instead of $a$.

In this paper, we derive an equation for the constant $a$ straight from equality (1) (equality (11) and another characterization of $a$ are derived in [6] rather implicitly).

2010 Mathematics Subject Classification. Primary 60G40; Secondary 60J65, 35 R35.

Key words and phrases. Optimal stopping, geometric Brownian motion, American option, a free boundary problem.

The author is indebted to the European Commission for support in the framework of the "Marie Curie Actions" program, grant PIRSES-GA-2008-230804. 


\section{THE DERIVATION OF AN EQUATION FOR THE CONSTANT $a$}

Consider equation (1) which defines the optimization problem for the standard Brownian motion. This equation is equivalent to

$$
\sup _{\tau \in[0,1]} \mathrm{E}\left[\tau\left(W_{\tau}-a\right)\right]=0
$$

Moreover, one can easily see that

$$
a=\sup \left\{c: \sup _{\tau \in[0,1]} \mathrm{E}\left[\tau\left(W_{\tau}-c\right)\right]=0\right\} .
$$

Thus it is natural to consider the following auxiliary optimization problem for $c \in \mathbb{R}$ :

$$
\mathrm{E}\left[\tau\left(W_{\tau}-c\right)\right] \rightarrow \max
$$

with respect to all stopping times $\tau \in[0,1]$.

The problem (2) is, in fact, a problem on the optimal exercise of an American type option. This allows us to use standard methods of the theory of estimation of American type contingent claims without going deep into detail (see, for example, [1, 7]).

Consider the following premium function,

$$
P(t, w)=P_{T, c}(t, w)=\sup _{\tau \in[t, T]} \mathrm{E}\left[\tau\left(W_{\tau}-c\right) \mid W_{t}=w\right]
$$

which represents the maximal expected continuation cost at the moment $t$ (which means that one does not exercise an option at the moment $t$ ) if the current value of the Brownian motion $W_{t}$ at the moment $t$ is equal to $w$. It is clear that the cost of stopping equals $t(w-c)$.

It is natural to define the stopping region for problem (2) by

$$
\mathcal{G}=\{(t, w) \in[0, T] \times \mathbb{R} \mid P(t, w) \leq t(w-c)\}
$$

and the continuation region by

$$
\mathcal{C}=\{(t, w) \in[0, T] \times \mathbb{R} \mid P(t, w)>t(w-c)\} .
$$

Then the (minimal) optimal stopping time for problem (2) is given by

$$
\tau^{*}=\inf \left\{t \in[0, T] \mid\left(t, W_{t}\right) \in \mathcal{G}\right\} .
$$

We agree that $\tau^{*}=T$ if the set on the right-hand side of the latter equality is empty.

Prior to continuing to solve the problem itself, we make a useful remark. Since the Brownian motion is homogeneous and possesses the scaling property,

$$
\begin{aligned}
P_{T, c}(t, w) & =\sup _{\tau \in[0, T-t]} \mathrm{E}\left[(\tau+t)\left(W_{\tau}+w-c\right)\right] \\
& =t(w-c)+(T-t)^{1 / 2} \sup _{\tau \in[0, T-t]} \mathrm{E}\left[\tau\left((T-t)^{-1 / 2} W_{\tau}+\widetilde{w}\right)\right] \\
& =t(w-c)+(T-t)^{3 / 2} \sup _{\tau \in[0,1]} \mathrm{E}\left[\tau\left(W_{\tau}+\widetilde{w}\right)\right] \\
& =t(w-c)+(T-t)^{3 / 2} P_{0,1}(0, \widetilde{w})=t(w-c)+(T-t)^{3 / 2} \psi(\widetilde{w}),
\end{aligned}
$$

where $\widetilde{w}=(w-c)(T-t)^{-1 / 2}$ and

$$
\psi(w)=P_{0,1}(0, w)=\sup _{\tau \in[0,1]} \mathrm{E}\left[\tau\left(W_{\tau}+w\right)\right] .
$$


It is clear that the function $\psi$ is nondecreasing, whence

$$
\begin{aligned}
\mathcal{G} & =\{(t, w) \mid P(t, w) \leq t(w-c)\} \\
& =\left\{(t, w) \mid t(w-c)+(T-t)^{3 / 2} \psi(\widetilde{w}) \leq t(w-c)\right\} \\
& =\{(t, w) \mid \psi(\widetilde{w}) \leq 0\}=\{(t, w) \mid \widetilde{w} \leq-a\} \\
& =\{(t, w) \mid w \leq c-a \sqrt{T-t}\} .
\end{aligned}
$$

Here

$$
a=-\sup \{x \mid \psi(x)=0\} .
$$

The latter constant coincides with the constant $a$ defined by equality (1).

Now we turn to the optimal stopping problem (21). As noted above, the optimal stopping time is the moment when the process

$$
X_{t}:=\left(t, W_{t}\right)
$$

exits the continuation region $\mathcal{C}$. Denote by

$$
\mathcal{L}=\frac{\partial}{\partial t}+\frac{1}{2} \frac{\partial^{2}}{\partial w^{2}}
$$

the generator of the diffusion process $X_{t}$. Then

$$
\mathcal{L} P=0 \text { in the region } \mathcal{C} .
$$

On the other hand,

$$
\mathcal{L} P=\mathcal{L}(t(w-c))=w-c, \quad(t, w) \in \mathcal{G},
$$

in the stopping region. Moreover, the following boundary conditions hold:

$$
\begin{gathered}
P(t, w)=t(w-c),(t, w) \in \partial \mathcal{C}, \\
\frac{\partial}{\partial w} P(t, w)=t,(t, w) \in \partial \mathcal{C}, \\
\frac{\partial}{\partial t} P(t, w)=w-c,(t, w) \in \partial \mathcal{C} .
\end{gathered}
$$

Equation (5) together with the "boundary conditions" (7)-(9) form the so-called "free boundary problem". We say that the problem is free boundary because conditions (7)-(9) are given at the boundary that is not known a priori but rather is determined from the equation itself. The above boundary conditions simply mean that the function $P$ is continuously differentiable and $P$ should be equal to $t(w-c)$ in the stopping region. In other words, we should fit smoothly a solution of (5) to the values in the stopping region.

We are able to solve the problem (more or less) explicitly for this simple setting. There are two approaches to solving this problem and they lead to different equations.

1. By Itô's formula,

$$
P\left(T, W_{T}\right)-P\left(t, W_{t}\right)=\int_{t}^{T} \mathcal{L} P\left(s, W_{s}\right) d s+\int_{t}^{T} \frac{\partial}{\partial w} P\left(s, W_{s}\right) d W_{s} .
$$


Consider the conditional expectation given $W_{t}=w$ :

$$
\begin{aligned}
& \mathrm{E}\left[P\left(T, W_{T}\right) \mid W_{t}=w\right]-P(t, w) \\
& \quad=\int_{t}^{T} \mathrm{E}\left[\mathcal{L} P\left(s, W_{s}\right) \mid W_{t}=w\right] d s \\
&=\int_{t}^{T} \mathrm{E}\left[\left(W_{s}-c\right) \mathbb{1}_{\left(s, W_{s}\right) \in \mathcal{G}} \mid W_{t}=w\right] d s \\
&=\int_{t}^{T} \mathrm{E}\left[\left(\left(W_{s}-w\right)+w-c\right) \mathbb{1}_{\left.W_{s} \leq c-a \sqrt{T-s} \mid W_{t}=w\right] d s}\right. \\
&=\int_{t}^{T} \mathrm{E}\left[(\sqrt{s-t} Z+w-c) \mathbb{1}_{\sqrt{s-t} Z+w \leq c-a \sqrt{T-s}] d s}\right. \\
&=(T-t) \int_{0}^{1} \mathrm{E}[(\sqrt{u(T-t)} Z+w-c) \mathbb{1} \sqrt{u(T-t)} Z+w-c \leq-a \sqrt{(1-u)(T-t)}] d u \\
&=(T-t)^{3 / 2} \int_{0}^{1} \mathrm{E}[\sqrt{u} Z+\widetilde{w}] \mathbb{1}_{\sqrt{u} Z+\widetilde{w} \leq-a \sqrt{1-u} d u,}
\end{aligned}
$$

where $Z$ is a standard normal random variable and $\widetilde{w}=(w-c)(T-t)^{-1 / 2}$. Denote by $\varphi$ and $\Phi$ the standard normal density and distribution function, respectively, and put $k(u)=-(\widetilde{w}+a \sqrt{1-u}) / \sqrt{u}$. Using the equality $P(T, w)=T(w-c)$, we obtain

$$
\begin{aligned}
T(w-c)-P(t, w) & =(T-t)^{3 / 2} \int_{0}^{1} \mathrm{E}[\sqrt{u} Z+\widetilde{w}] \mathbb{1}_{\sqrt{u} Z \leq-\widetilde{w}-a \sqrt{1-u} d u} \\
& =(T-t)^{3 / 2} \int_{0}^{1}[-\sqrt{u} \varphi(k(u))+\widetilde{w} \Phi(k(u))] d u .
\end{aligned}
$$

Now we put $w=c-a \sqrt{T-t}$. Note that $(t, w) \in \partial C$, whence $P(t, w)=t(w-c)$. Recalling that $\widetilde{w}=(w-c)(T-t)^{-1 / 2}$, we deduce that $\widetilde{w}=-a$ in this case. Hence equation (10) is reduced to

$$
-a=\int_{0}^{1}[-\sqrt{u} \varphi(a \mu(u))-a \Phi(a \mu(u))] d u,
$$

where $\mu(u)=(1-\sqrt{1-u}) / \sqrt{u}$. Therefore

$$
a=\int_{0}^{1} \sqrt{u} \varphi(-a \mu(u)) d u / \int_{0}^{1} \Phi(-a \mu(u)) d u .
$$

This equation is derived in the paper [5], too (however the setting in [5] differs essentially from the one in this paper). It is also proved in 5 , that the latter equation has a unique positive solution belonging to the interval $\left[0,2^{-1 / 2}\right)$.

2. Another idea is to use property (3) and substitute it into equation (4). After simple algebra we get

$$
\mathcal{L} P(t, w)=w-c-\frac{3}{2}(T-t)^{1 / 2} \psi(\widetilde{w})+\frac{1}{2}(w-c) \psi^{\prime}(\widetilde{w})+\frac{1}{2}(T-t)^{1 / 2} \psi^{\prime \prime}(\widetilde{w}),
$$

where $\widetilde{w}$ is the same as above.

Thus the equation $\mathcal{L} P(t, w)=0$ for $(t, w) \in \mathcal{C}$ is equivalent to

$$
\psi^{\prime \prime}(x)+x \psi(x)-3 \psi(x)=-2 x
$$

for $x>a$. The general solution to the latter equation is given by

$$
\psi(x)=-\frac{x^{3}}{3}+C_{1} x\left(3+x^{2}\right)+C_{2}\left(\left(2+x^{2}\right) \varphi(x)-x\left(3+x^{2}\right) \Phi(-x)\right) .
$$


Since $P(T, w)=T(w-c)$, we get $\psi(\widetilde{w})(T-t)^{3 / 2} \rightarrow 0$ as $t \rightarrow T$ or, equivalently, $\psi(x) / x^{3} \rightarrow 0$ as $x \rightarrow \pm \infty$. Passing to the limit in (12) as $x \rightarrow+\infty$ we get $C_{1}=1 / 3$, whence

$$
\psi(x)=x+C_{2}\left(\left(2+x^{2}\right) \varphi(x)-x\left(3+x^{2}\right) \Phi(-x)\right) .
$$

Further, we reduce condition (77) to $\psi(-a)=0$ and similarly conditions (8) and (9) to $\psi^{\prime}(-a)=0$. Therefore $\psi(-a)+a \psi^{\prime}(-a)=0$, whence

$$
C_{2}\left(\left(a^{2}-1\right) \varphi(a)+a^{3} \Phi(a)\right)=0 .
$$

Note that $C_{2} \neq 0$ (otherwise $a=0$ and the latter equality does not hold). Now we obtain the equation

$$
\left(1-a^{2}\right) \varphi(a)=a^{3} \Phi(a) .
$$

A similar equation, but with $a \sqrt{2}$ instead of $a$, is also derived in 8 .

In conclusion we note that, first, the constant $a$ appears in problems of an optimal exercise of a future contract for selling an asset for a predetermined price. It turns out that this problem is equivalent to the above-mentioned problem of optimal exchange of assets; see [6. Second, solving both equations (11) and (13) numerically, we obtain the same value of the approximate solution, namely $a=0.6388332158 \ldots$.

\section{BIBLIOGRAPHY}

1. M. Broadie and J. Detemple, The valuation of American options on multiple assets, Math. Finance 7 (1997), no. 3, 241-286. MR.1459060 (98b:90012)

2. M. Ehrhardt and R. E. Mickens, A fast, stable and accurate numerical method for the BlackScholes equation of American options, Int. J. Theor. Appl. Finance 11 (2008), no. 5, 471-501. MR 2450224 (2009f:91055)

3. J. Evans, R. Kuske, and J. B. Keller, American options on assets with dividends near expiry, Math. Finance 12 (2002), no. 3, 219-237. MR.1910594 (2003e:91079)

4. P. V. Johnson, N. J. Sharp, P. W. Duck, and D. P. Newton, A new class of option: the American delayed-exercise option, VIII Encontro Brasileiro de Finanças, Rio de Janeiro, 2008.

5. D. Lamberton and S. Villeneuve, Critical price near maturity for an American option on a dividend-paying stock, Ann. Appl. Probab. 13 (2003), no. 2, 800-815. MR1970287 (2004d:91116)

6. Y. Mishura and G. Shevchenko, The optimal time to exchange one asset for another on finite interval, Optimality and Risk - Modern Trends in Mathematical Finance. The Kabanov Festschrift (Delbaen, Freddy et al., eds.), Springer, Berlin, 2009, pp. 197-210. MR2648604 (2011h:60096)

7. S. Villeneuve, Exercise regions of American options on several assets, Finance Stoch. 3 (1999), no. 3, 295-322.

8. P. Wilmott, S. Howison, and J. Dewynne, The Mathematics of Financial Derivatives. A Student Introduction, Cambridge Univ. Press, Cambridge, 1995. MR.1357666 (96h:90028)

Department of Probability Theory, Statistics, and Actuarial Mathematics, Faculty for Mechanics and Mathematics, National Taras Shevchenko University, Academician Glushkov Avenue 2, Kiev 03127, Ukraine

E-mail address: zhora@univ.kiev.ua

Received 22/FEB/2010

Translated by S. KVASKO 